\title{
Tm7sf2 may participate in the healing of burn wounds
}

\author{
$\mathrm{NALEI}^{1 *}, \mathrm{PENG} \mathrm{WANG}^{2 *}$ and QIANG MENG ${ }^{3}$ \\ ${ }^{1}$ Department of Burn and Plastic Surgery, First Affiliated Hospital of Shihezi University Medical College, Shihezi, \\ Xinjiang 832008; ${ }^{2}$ Deparment of Plastic and Aesthetic Center, Yantai Yuhuangding Hospital, Yantai Shandong 264000; \\ ${ }^{3}$ Department of Intensive Care Unit, Jining First People's Hospital, Jining, Shandong 272011, P.R. China
}

Received May 12, 2015; Accepted January 8, 2016

DOI: $10.3892 / \mathrm{mmr} .2016 .5310$

\begin{abstract}
The transmembrane 7 superfamily member 2 (Tm7sf2) gene been reported to be involved in the reduced cholesterollevels of patients withlarge burn areas. Toinvestigate the importance of Tm7sf 2 in the burn wound healing process, a total of 10 Sprague-Dawley rats underwent electrical burns. Blood serum was collected for the culture of HaCaT human keratinocyte cells. Tm7sf 2 small interfering RNAs (siRNAs) were prepared and transfected into the normal- and burn serum-cultured HaCaT cells. Monocyte-endothelial cellular adhesion ability and cell proliferation, as determined by 3-(4,5-dimethylthiazol-2-yl)-2,5-diphenyltetrazolium bromide assay, and the levels of autophagy proteins Beclin1 and LC3-II, which were detected by western blot assay and real time-quantitative polymerase chain reaction, and it was identified that Tm7sf2 siRNAs were successfully transfected. Cell proliferation was reduced and monocyte-endothelial cell adhesion increased in the burn serum group compared with the normal serum group $(\mathrm{P}<0.05)$. Additionally, the expression levels of Beclin1 and LC3-II were increased, and the addition of siTm7sf 2 had a similar effect as the burn serum. Thus, Tm7sf 2 may take part in the burn wound healing process by interacting with LC3-II and Beclin1, and targeting Tm7sf 2 may have therapeutic benefits.
\end{abstract}

\section{Introduction}

Burn injury is one of the most common and severe trauma morphologies and is typically established from thermal injury of the skin (1). The clinical features of burn injuries range from difficult initial assessment to the tendency of pathological scars (2). According to a previous 33-year

Correspondence to: Mr. Qiang Meng, Department of Intensive Care Unit, Jining First People's Hospital, 6 Jiankang Road, Jining, Shandong 272011, P.R. China

E-mail: mengqiang985@126.com

*Contributed equally

Key words: wound healing, transmembrane 7 superfamily member 2, mechanism, LC3-II, Beclin 1, autophagy population-based study, adjusted for all-cause mortality, the mortality rate of burn injury was 1.6 (95\% confidence interval, 1.3-2.0), and children with burn injuries had a 1.6-fold higher mortality rate than those without any injuries (3). The resuscitation of burn trauma patients remains a challenge, as the healing mechanism remains to be fully elucidated. When treating burn wounds it is crucial to restore and close the exposed wound surface promptly, as this will aid in limiting the loss of water, electrolytes and nutrients, and preventing the invasion of pathogenic microorganisms (4-6). Aspects of the wound healing mechanism remain unclear; however, it has been established that multiple cell types, the extracellular matrix and soluble mediators are all involved in this process (7).

The transmembrane 7 superfamily member $2(\mathrm{Tm} 7 \mathrm{sf} 2)$ gene has been reported to be involved in cholesterol biosynthesis by encoding the protein $3 \beta$-hydroxysterol $\Delta 14$-reductase (8). Tm7sf 2 participates in the cellular response to stressful conditions (9) and its loss alters the expression of proteins that are involved in the differentiation of epidermal cells by reducing cholesterol sulfate levels (10). The loss of the entire epidermis following burn injury may induce severe hematopoietic dysfunction, which will subsequently increase the risk of mortality and morbidity (11). A previous study indicated that cholesterol levels of the erythrocyte membrane in patients with large area burns were significantly increased. Therefore, it is possible that Tm7sf2 may be involved in the wound healing process (12).

Electrical burns at $1,000 \mathrm{~V}$ may result in extensive damage to tissues and even renal failure or rhabdomyolysis (13). The current study performed electrical burns on rats. The burn serum was then extracted and used to culture HaCaT human keratinocyte cells were. Tm7sf2 siRNAs were also produced to determine their effect on these cells. Cell proliferation, monocyte-endothelial cell adhesion ability and the expression levels of the autophagy-associated proteins, Beclin1 and LC3-II, were additionally investigated, in order to determine whether Tm7sf2 participates in the process of burn wound healing.

\section{Materials and methods}

Burn wound model. Sprague-Dawley rats (age, 5 weeks old; male and female; $\mathrm{n}=10$ ) weighing between 250 and $300 \mathrm{~g}$ and maintained under controlled temperature $\left(20 \pm 1^{\circ} \mathrm{C}\right)$, humidity $(55 \pm 10 \%)$ and illumination (12 h dark:light), were subjected to limb electrical burns as described previously (14). Subsequent 
to undergoing anesthesia with $3 \%$ pentobarbital $(10 \mathrm{mg} / \mathrm{kg}$; Sigma-Aldrich, St. Louis, MO, USA), the rats were maintained in a stationary position on the experimental table in order to shave the hip and right posterior limb. Next, a null electrode was placed on the right limb $(0.5 \mathrm{~cm}$ proximal to ankle), with a live electrode placed on the ipsilateral gluteus. The electricity (output voltage: 1,000 V) was administered for $0.1 \mathrm{sec}$ to conduct the burns using the YLS-5Q Skin Burning Device model (Biowill Co, Ltd. Shanghai, China). Blood samples were obtained from the tail vein (using $1 \mathrm{ml}$ syringe) at 6 and 24 and $36 \mathrm{~h}$, subsequent to the administration of the electric burns, and then centrifuged at $300 \times \mathrm{g},-4^{\circ} \mathrm{C}$ for $15 \mathrm{~min}$, to obtain the blood serum.

Cell culture. HaCaT cells (Shanghai Bogoo Biotechnology Co., Ltd., Shanghai, China) were cultured in Dulbecco's modified Eagle's medium (DMEM; Gibco; Thermo Fisher Scientific, Inc., Waltham, MA, USA) medium supplemented with $10 \%$ (v/v) fetal bovine serum, $100 \mathrm{U} / \mathrm{ml}$ streptomycin and $100 \mathrm{U} / \mathrm{ml}$ penicillin at $37^{\circ} \mathrm{C}$ in a $5 \%(\mathrm{v} / \mathrm{v}) \mathrm{CO}_{2}$ humidified atmosphere. DMEM (Gibco; Thermo Fisher Scientific, Inc.) containing $0.5 \mathrm{mg} / \mathrm{ml}$ collagenase type I was applied at $37^{\circ} \mathrm{C}$ for $3 \mathrm{~h}$ to separate fibroblasts from rat blood serum. These fibroblasts were pelleted by centrifugation at $300 \mathrm{x} \mathrm{g}$, for $7 \mathrm{~min}$, at room temperature, and then cultured in the aforementioned conditions. Cells passaged 2-4 times were used for further experiments.

$T m 7 s f 2$ transfection. The full-length wild-type Tm7sf2 coding sequence (alternate names: D14SR, SR-1; RefSeq accession, NP_003264) was sub-cloned into pcDNA3.1 $1^{+}$to produce the Tm7sf2 expression vector (pcDNA3.1- Tm7sf2), which was confirmed by sequencing at Shanghai Majorbio Bio-pharm Technology Co., Ltd. (Shanghai, China). Tm7sf2-specific siRNA and control siRNA were synthesized by Shanghai GenePharma Co., Ltd. (Shanghai, China). Lipofectamine 2000 reagent (Invitrogen; Thermo Fisher Scientific, Inc.) was used for the cell transfection, according to manufacturer's protocol, and empty construct pcDNA3.1 was also transfected as a control. Stable Tm7sf2 transfectants were selected using G418 (Gibco; Thermo Fisher Scientific, Inc.).

Cell interference experiments. HaCaT cells were divided into four groups and exposed to the following treatments for $20 \mathrm{~h}$ : i) Control, $20 \%$ normal serum; ii) burn, $20 \%$ electrical burned serum; iii) $20 \%$ normal serum + siTm7sf2; iv) $20 \%$ electrical burned serum + siTm7sf2.

Cell proliferation and invasiveness. A 3-(4,5-dimethylthiazol-2-yl)-2,5-diphenyltetrazolium bromide (MTT; Sigma-Aldrich) colorimetric assay and Matrigel (BD Biosciences, Franklin Lakes, NJ, USA) invasion chamber assay were used for the determination of cell proliferative and invasive capacities, respectively, as described previously $(15,16)$. Each experiment was performed three times.

Monocyte-endothelial cell adhesion assay. Calcein-acetomethyl (25 mg; Molecular Probes; Thermo Fisher Scientific, Inc., in $5 \mathrm{ml}$ dimethyl sulfoxide/ml media; Sigma-Aldrich) was used for labeling the treated HaCaT cells. Subsequent to
Table I. Tm7sf microRNA and protein expression levels.

\begin{tabular}{lcc}
\hline Time (h)/group & mRNA level & Protein level \\
\hline 0 & $1.00 \pm 0.00$ & - \\
6 & $1.18 \pm 0.07$ & - \\
12 & $1.32 \pm 0.05$ & - \\
24 & $1.49 \pm 0.09$ & - \\
36 & $1.25 \pm 0.05$ & $1.00 \pm 0.00$ \\
Control & - & $1.43 \pm 0.11$ \\
Tm7sf2 & - & $0.51 \pm 0.09$ \\
siTm7sf2 & - & $1.03 \pm 0.12$ \\
Tm7sf2+siTm7sf2 & - &
\end{tabular}

mRNA and protein expression levels are presented as the fold-changes from the $0 \mathrm{~h}$ and control groups, respectively. Tm7sf2, transmembrane 7 superfamily member 2 ; si, small interfering.

centrifugation at $300 \mathrm{xg}$, for $7 \mathrm{~min}$, at room temperature, the cells were washed three times with phosphate-buffered saline (PBS; Sigma-Aldrich) to allow the adherence to a compact monolayer of the EA.hy926 human endothelial cell line (Cell bank of Type Culture Collection of the Chinese Academy of Sciences, Shanghai, China) at $37^{\circ} \mathrm{C}$ for $1 \mathrm{~h}$. Subsequently, the monocytes in the medium were aspirated. A fluorescence microscope (Olympus CX31; Olympus Corporation, Tokyo, Japan) was used for the visualization cell adherence at excitation and emission wavelengths of 488 and $535 \mathrm{~nm}$, respectively. Six fields were selected per well to calculate the number of adherent monocytes using Image $\mathbf{J}$ software (version 2; National Institutes of Health, Bethesda, MD, USA).

Reverse transcription-quantitative polymerase chain reaction $(R T-q P C R)$. RT-qPCR was performed as previously described (17). Total mRNA was isolated from cells, and complementary DNA (cDNA) was produced using reverse transcriptase (iScript cDNA Synthesis kit; Bio-Rad Laboratories, Inc., Hercules, CA USA). Tm7sf2 expression levels were measured via SYBR-Green based qPCR (SYBR Green Master mix; Thermo Fisher Scientific, Inc.). Reactions were performed with the initial denaturation at $95^{\circ} \mathrm{C}$ for $5 \mathrm{~min}$ and then $40-50$ cycles at $95^{\circ} \mathrm{C}$ for $30 \mathrm{sec}, 58-62^{\circ} \mathrm{C}$ for $30 \mathrm{sec}$ and $72^{\circ} \mathrm{C}$ for $30 \mathrm{sec}$, and a final extension at $72^{\circ} \mathrm{C}$ for $10 \mathrm{sec}$.

Western blot analysis. A 10-12\% sodium dodecyl sulfate-polyacrylamide gel (Thermo Fisher Scientific, Inc.) was used for protein separation at $100 \mathrm{~V}$ for $90 \mathrm{~min}$. Next, the proteins were blotted onto polyvinylidene difluoride membranes, which were blocked with PBST (0.1\% Triton X-100; Sigma-Aldrich in PBS), and probed with primary antibodies including anti-Tm7sf2 (cat no. sc-162325) Beclin 1 (cat no. sc-11427) and LC3-II (cat no. sc-28266) all diluted 1:200 in bovine serum albumin) and horseradish peroxidase-conjugated secondary antibodies (sc-391122; diluted 1:5,000) and GAPDH (sc-595401; diluted 1:1,000). GAPDH was used as a loading control. All antibodies were purchased from Santa Cruz Biotechnology, Inc., (Dallas, TX, USA) unless otherwise stated. Enhanced chemiluminescence (GE Healthcare Life Sciences; Uppsala, Sweden) was 
Table II. Proliferation of HaCaT cells .

\begin{tabular}{lcc}
\hline Group & siTm7sf & Absorbance level (Abs) \\
\hline Normal serum & - & $1.03 \pm 0.01$ \\
& + & $0.74 \pm 0.05$ \\
Burn serum & - & $0.78 \pm 0.06$ \\
& + & $0.56 \pm 0.11$
\end{tabular}

siTm7sf2, small interfering transmembrane 7 superfamily member 2 .

A

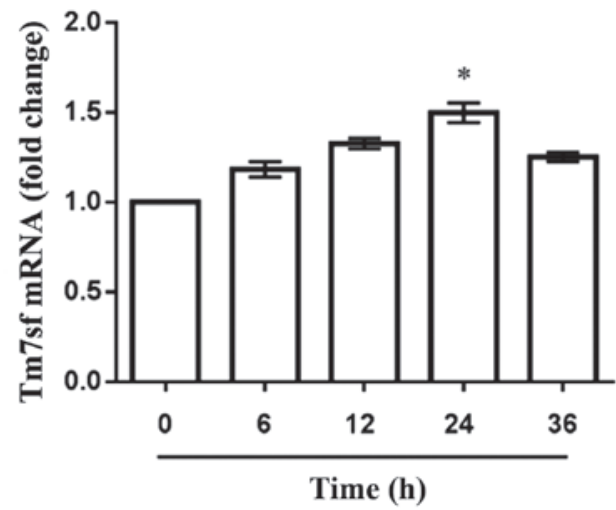

B
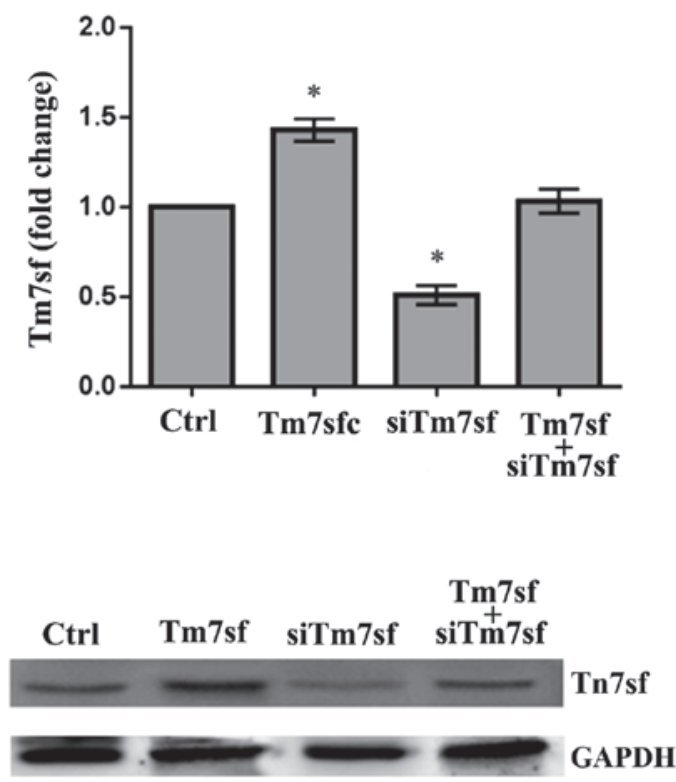

Figure 1. Tm7sf2 levels. (A) mRNA expression levels in rats with electric burns. (B) Protein expression levels in $\mathrm{HaCaT}$ cells. ${ }^{*} \mathrm{P}<0.05$ vs. control (no burns). Tm7sf2, transmembrane 7 superfamily member 2 ; si, small interfering; GAPDH, glyceraldehyde 3-phosphatase dehydrogenase.

utilized to develop the immunoreactive protein bands, which were analyzed by a densitometer (Image Pro Plus, version 1.63; Media Cybernetics, Rockville, MD, USA).

Statistical analysis. Each sample was processed and measured three times. The data are presented as the mean \pm standard
Table III. Numbers of monocytes.

\begin{tabular}{lcc}
\hline Group & siTm7sf & No. \\
\hline Normal serum & - & $209.67 \pm 9.50$ \\
\multirow{2}{*}{ Burn serum } & + & $438.00 \pm 33.65$ \\
& - & $606.67 \pm 43.32$ \\
& + & $680.67 \pm 24.58$
\end{tabular}

siTm7sf2, small interfering transmembrane 7 superfamily member 2 .

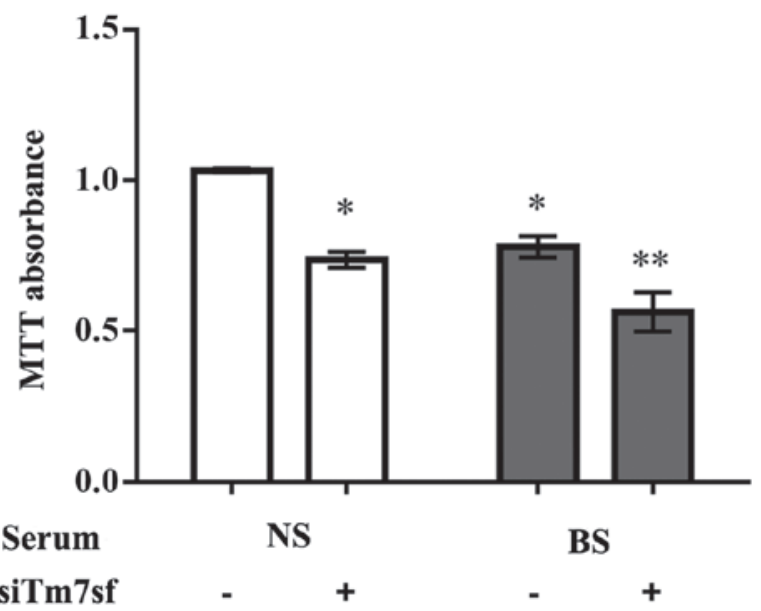

Figure 2. Proliferation of $\mathrm{HaCaT}$ cells. ${ }^{*} \mathrm{P}<0.05,{ }^{* *} \mathrm{P}<0.01$ vs. control (no burns). NS, normal serum treated; BS, burn serum; MTT, 3-(4,5-dimethylthiazol-2-yl)-2,5-diphenyltetrazolium bromide.

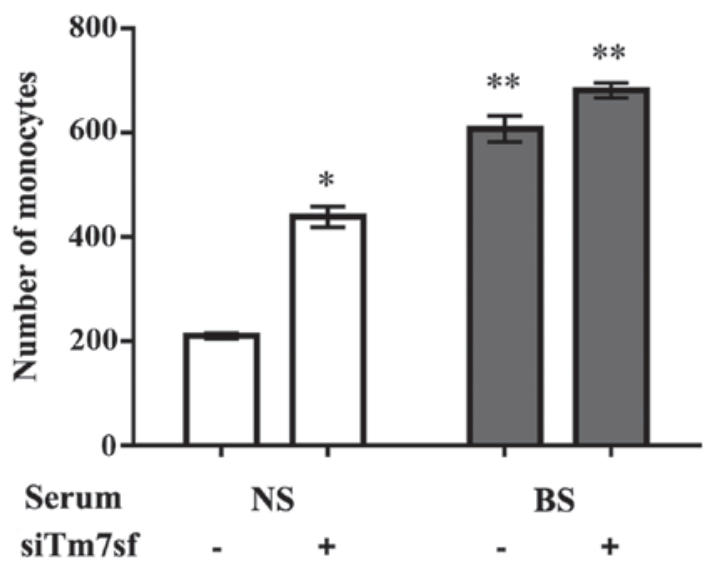

Figure 3. Monocyte-endothelial cell adhesion between $\mathrm{HaCaT}$ and EA.hy 926 cells. ${ }^{*} \mathrm{P}<0.05,{ }^{* *} \mathrm{P}<0.01$ vs. control (no burns). NS, normal serum treated; BS, burn serum; siTm7sf2, small interfering transmembrane 7 superfamily member 2 .

deviation. The statistical analysis was performed using SPSS software (version 17.0; SPSS, Inc., Chicago, IL, USA) statistical software, one-way analysis of variance, followed by Fisher's Protected Least Significant Difference post-hoc 
Table IV. Beclin1 and LC3-II protein expresion levels in HaCaT cells.

\begin{tabular}{lccc}
\hline Group & siTm7sf & Beclin1 & LC3-II \\
\hline Normal serum & - & $1.03 \pm 0.01$ & $1.78 \pm 0.06$ \\
& + & $1.65 \pm 0.16$ & $2.20 \pm 0.25$ \\
Burn serum & - & $82.43 \pm 10.67$ & $157.45 \pm 16.11$ \\
& + & $128.32 \pm 13.23$ & $192.87 \pm 12.12$
\end{tabular}

siTm7sf2, small interfering transmembrane 7 superfamily member 2 .

analysis used to determine statistical differences between groups. $\mathrm{P}<0.05$ was considered to indicate a statistically significant difference.

\section{Results}

$T m 7 s f 2$ level detection. Compared with the control group, Tm7sf2 mRNA levels in rats with electric burns were significantly higher with the increasing time following the administration of the burn $(\mathrm{P}<0.05)$. The levels were then reduced after $24 \mathrm{~h}$ (Fig. 1A). In the $20 \%$ electrical burned serum group, the protein levels of $\mathrm{Tm} 7 \mathrm{sf} 2$ were significantly higher than the control group $(\mathrm{P}<0.05)$. In the siTm7sf2-transfected cells, Tm7sf2 expression was significantly lower compared with the control group $(\mathrm{P}<0.05)$, however was restored to normal with the addition of Tm7sf 2 (Fig. 1B; Table I).

Cell proliferation. The MTT assay demonstrated that the burn serum significantly reduced cell proliferation when compared with the normal serum $(\mathrm{P}<0.05)$. Furthermore the proliferation of cells treated with Tm7sf 2 siRNA was significantly lower in the burn serum group compared with the control cells $(\mathrm{P}<0.05$; Fig. 2; Table II).

Monocyte-endothelial cell adhesion. The number of monocytes represent the adhesion strength between the EA.hy 926 and $\mathrm{HaCaT}$ cells (18). The $24 \mathrm{~h}$ treatment with burn serum induced the highest expression levels of Tm7sf2, therefore cell adhesion of the HaCaT cells was detected subsequent to $24 \mathrm{~h}$ of treatment. The burn serum treatment significantly enhanced the capacity of monocyte-endothelial cell adhesion, even if the cells had been transfected with siTm7sf2, compared with normal serum treatment $(\mathrm{P}<0.05$; Fig. 3 ; Table III).

Autophagy-associated protein levels. To investigate the influence of the burn serum and siTm7sf 2 on autophagic abilities of $\mathrm{HaCaT}$ cells, the autophagy-associated Beclin1 and LC3-II protein levels in $\mathrm{HaCaT}$ cells were detected using western blot analysis. As indicated in Fig. 4, burn serum (treated for $24 \mathrm{~h}$ ) increased the expression levels of Beclin1 and LC3-II when compared with control groups $(\mathrm{P}<0.05)$. The siTm7sf2 group also presented increased expression levels, while the effect was more evident in the burn serum-treated cells $(\mathrm{P}<0.01$; Table IV).
$\mathbf{A}$

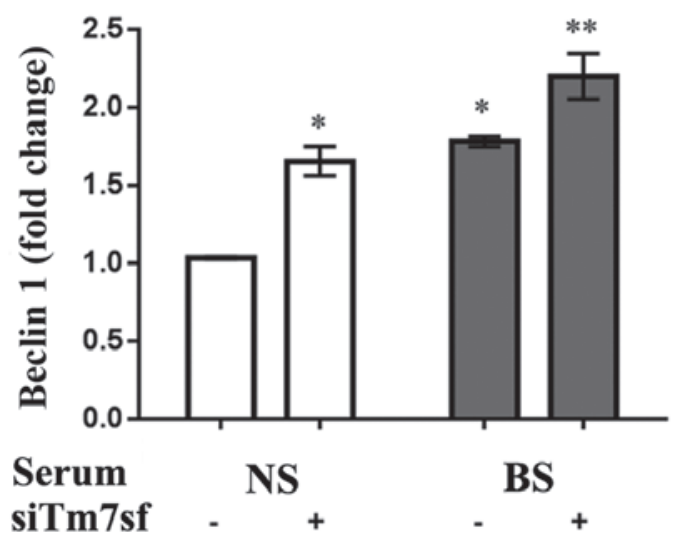

B
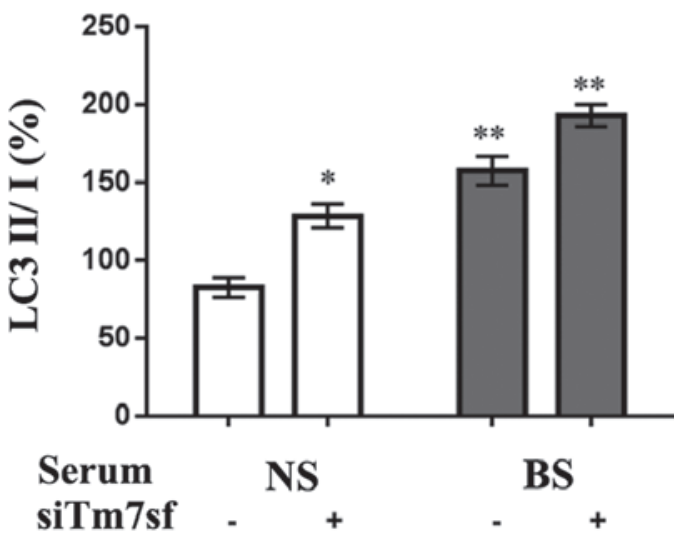

C

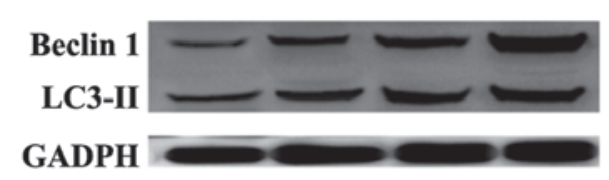

Figure 4. (A) Beclin1 and (B) LC3-II protein expression levels in HaCaT cells. (C) Western blotting of protein expression levels. ${ }^{*} \mathrm{P}<0.05$, ${ }^{* *} \mathrm{P}<0.01$ vs. the controls (no burns). NS, normal serum treated; BS, burn serum; siTm7sf2, small interfering transmembrane 7 superfamily member 2 ; GAPDH, glyceraldehyde 3-phosphatase dehydrogenase.

\section{Discussion}

In the current study, it was identified that burn serum treatment reduced cell proliferation, increased monocyte-endothelial cell adhesion, and induced higher expression levels of Beclin1 and LC3-II in HaCaT cells. Additionally, the administration of siTm7sf 2 had a similar influence on these properties, suggesting that $T \mathrm{~m} 7 \mathrm{sf} 2$ may be important during burn wound repair.

Thermal injury induces the release of inflammatory factors (19). Monocyte-endothelial cellular adhesion, as a key feature in inflammation, is important in monocyte extravasation (14). The observations of the present study indicate that silencing of Tm7sf 2 and treatment with burn serum increase cellular adhesion, with the siTm7sf 2 + burn serum treatment resulting in the highest number of monocytes. Therefore, 
Tm7sf 2 may be involved in inflammation during the wound healing process.

Autophagy is a catabolic process necessary for the maintenance of the cellular organelle balance, and is a highly conserved pathway for the delivery of intracellular macromolecule waste to lysosomes (20). Severe burns lead to endoplasmic reticulum stress that triggers autophagy signaling cascades. However, it has been identified that there is reduction in autophagy in burn wounds during the initial period of progression of burn injuries (21). The enhanced level of autophagy deep in the dermis has been suggested as a potential prosurvival factor against injury-associated inflammation (22). The Beclin1 protein is associated with the activation of autophagy and in addition, as a reliable biomarker of autophagy activity, it forms part of the phosphatidylinositol 3-kinase complex (23). LC3 is a critical protein for autophagy, and its cleavage leads to the production of LC3-I, which is converted to LC3-II subsequent to lipidation via a ubiquitin-like system that allows LC3 to be involved in autophagic vesicles (24). The LC3-II/LC3-I ratio is considered to be an indicator of autophagy (25). Furthermore, the increased expression levels of Beclin1 and LC3-II, two indicators of autophagy, observed in the current study suggested that the cells were undergoing the wound healing processes. Tm7sf2 also appears to be crucial to these processes.

In conclusion, burn serum extracted from an electrical burn rat model increased the expression of Tm7sf 2 . In addition, Tm7sf2 may participate in the wound healing process by interacting with LC3-II and Beclin1 and thus, therapeutically targeting Tm7sf 2 may be advantageous to the wound healing process following burn injuries. However, only rat models were used in the present study regarding the role of Tm7sf 2 , and further clinical or human tissue based studies are required to determine the benefit of novel therapies targeting Tm7sf2 for burn wound healing.

\section{References}

1. Weber $\mathbf{J}$ and McManus A; Nursing Committee of the International Society for Burn Injuries: Infection control in burn patients. Burns 30: 16-24, 2004

2. Teot L, Otman S, Brancati A and Mittermayr R (eds): Burn wound healing: Pathophysiology. In: Handbook of Burns. Vol 2. 1st edition. Springer Vienna, pp47-54, 2012.

3. Duke JM, Rea S, Boyd JH, Randall SM and Wood FM: Mortality after burn injury in children: A 33-year population-based study. Pediatrics 135: e903-e910, 2015.

4. Enoch S and Leaper DJ: Basic science of wound healing. Surgery 26: 31-37, 2008.

5. Li J, Chen J and Kirsner R: Pathophysiology of acute wound healing. Clin Dermatol 25: 9-18, 2007.

6. Velnar T, Bailey T and Smrkolj V: The wound healing process: An overview of the cellular and molecular mechanisms. J Int Med Res 37: 1528-1542, 2009.
7. Gurtner GC, Werner S, Barrandon Y and Longaker MT: Wound repair and regeneration. Nature 453: 314-321, 2008 (Review).

8. Zuleger N, Boyle S, Kelly DA, de las Heras JI, Lazou V, Korfali N, Batrakou DG, Randles KN, Morris GE, Harrison DJ, et al: Specific nuclear envelope transmembrane proteins can promote the location of chromosomes to and from the nuclear periphery. Genome Biol 14: R14, 2013.

9. Bellezza I, Roberti R, Gatticchi L, Del Sordo R, Rambotti MG Marchetti MC, Sidoni A and Minelli A: A novel role for Tm7sf2 gene in regulating TNFa expression. PLoS One 8: e68017, 2013

10. Bellezza I, Gatticchi L, del Sordo R, Peirce MJ, Sidoni A, Roberti R and Minelli A: The loss of Tm7sf gene accelerates skin papilloma formation in mice. Sci Rep 5: 9471, 2015.

11. Loebl EC, Baxter CR and Curreri PW: The mechanism of erythrocyte destruction in the early post-burn period. Ann Surg 178: 681-686, 1973.

12. Dalal R, Sharma CA, Chakravarty BB, Alam Parwaz CM and Anil Malik C: A study of prognostic factors for prediction of complications and outcomes in burn patients. Indian J Burns 22: 56-61, 2014.

13. Benson A and Dickson WA: Burns. ABC of wound healing BMJ 332: 649-652, 2006.

14. Ruan Q, Zhao C, Ye Z, Ruan J, Xie Q and Xie W: Effect and possible mechanism of monocyte-derived VEGF on monocyte-endothelial cellular adhesion after electrical burns. Burns 41: 825-832, 2015.

15. Zhang T, Liu M, Wang C, Lin C, Sun Y and Jin-D: Down-regulation of MiR-206 promotes proliferation and invasion of laryngeal cancer by regulating VEGF expression. Anticancer Res 31: 3859-3863, 2011.

16. Yang LH, Xu HT, Han Y, Li QC, Liu Y, Zhao Y, Yang ZQ, Dong QZ, Miao Y, Dai SD and Wang EH: Axin downregulates TCF-4 transcription via beta-catenin, but not p53, and inhibits the proliferation and invasion of lung cancer cells. Mol Cancer 9: $25,2010$.

17. Fransson L, Rosengren V, Saha TK, Grankvist N, Islam T, Honkanen RE, Sjöholm Å and Ortsäter H: Mitogen-activated protein kinases and protein phosphatase 5 mediate glucocorticoid-induced cytotoxicity in pancreatic islets and $\beta$-cells. Mol Cell Endocrinol 383: 126-136, 2014.

18. Edwards AM, Potter U, Meenan NA, Potts JR and Massey RC: Staphylococcus aureus keratinocyte invasion is dependent upon multiple high-affinity fibronectin-binding repeats within FnBPA. PLoS One 6: e18899, 2011.

19. Zhu H, Ka B and Murad F: Nitric oxide accelerates the recovery from burn wounds. World J Surg 31: 624-631, 2007.

20. Shintani T and Klionsky DJ: Autophagy in health and disease: A double-edged sword. Science 306: 990-995, 2004.

21. Song J, de Libero J and Wolf SE: Hepatic autophagy after severe burn in response to endoplasmic reticulum stress. J Surg Res 187: 128-133, 2014.

22. Xiao M, Li L, Li C, Zhang P, Hu Q, Ma L and Zhang H: Role of autophagy and apoptosis inwound tissue of deep second-degree burn in rats. Acad Emerg Med 21: 383-391, 2014.

23. Liang XH, Jackson S, Seaman M, Brown K, Kempkes B, Hibshoosh $\mathrm{H}$ and Levine B: Induction of autophagy and inhibition of tumorigenesis by beclin 1. Nature 402: 672-676, 1999.

24. Ichimura Y, Kirisako T, Takao T, Satomi Y, Shimonishi Y, Ishihara N, Mizushima N, Tanida I, Kominami E, Ohsumi M, et al: A ubiquitin-like system mediates protein lipidation. Nature 408: 488-492, 2000.

25. Kabeya Y, Mizushima N, Yamamoto A, Oshitani-Okamoto S, Ohsumi Y and Yoshimori T: LC3, GABARAP and GATE16 localize to autophagosomal membrane depending on form-II formation. J Cell Sci 117: 2805-2812, 2004. 